\title{
Moderate degradation does not preclude microarray analysis of small amounts of RNA
}

\author{
Oliver Schoor, Toni Weinschenk, Jörg Hennenlotter, Stefan Corvin, Arnulf Stenzl, Hans-Georg \\ Rammensee, and Stefan Stevanović
}

BioTechniques 35:1192-1201 (December 2003)

\begin{abstract}
Gene expression analysis by microarrays using small amounts of RNA is becoming more and more popular against the background of advances and increasing importance of small-sample acquisition methods like laser microdissection techniques. The quality of RNA preparations from such samples constitutes a frequent issue in this context. The aim of this study was to assess the impact of different extents of RNA degradation on the expression profile of the samples. We induced RNA degradation in human tumor and healthy tissue samples by endogeneous ribonucleases. Next, we amplified 20 ng total RNA degraded to different extents by two rounds of in vitro transcription and analyzed them using Affymetrix oligonucleotide microarrays. Expression differences for some genes were independently confirmed by real-time quantitative PCR. Our results suggest that gene expression profiles obtained from partially degraded RNA samples with still visible ribosomal bands exhibit a high degree of similarity compared to intact samples and that RNA samples of suboptimal quality might therefore still lead to meaningful results if used carefully.
\end{abstract}

\section{INTRODUCTION}

Since the initial days of gene expression analysis by DNA microarrays, this technique has become more and more a standard tool for many research groups in all areas of life sciences. The possibility of measuring the expression of thousands of genes simultaneously under different conditions has led to new insights into the behavior of various biological systems (1) and has provided new opportunities with respect to diagnosis or classification of diseases (2).

Recent advances in RNA and cDNA amplification methods have enabled microarray studies on small samples right down to very few cells $(3,4)$. One of the most commonly applied methods for small-sample amplification is based on a technique originally described by the group of Eberwine and coworkers (5). The basic steps involve reverse transcription of mRNA with an oligo(dT) primer containing a promoter sequence for T7 RNA polymerase. Synthesis of double-stranded cDNA is followed by an in vitro transcription (IVT) reaction resulting in multiple copies of antisense RNA (aRNA) from each cDNA molecule. Variations of this protocol enable its repetition in a second or even additional rounds and, therefore, qualify it for the synthesis of sufficient amounts of aRNA or cDNA for microarray analysis from only a few nanograms of total RNA. Even though such IVT-based methods have been widely used, especially in combination with laser microdissection (6-9), it has only recently been demonstrated by extensive evaluations that they can indeed be applied without introducing too much artificial bias (10-16).

One important issue, in the context of small-sample microarray analysis, in particular, remains the quality of initial RNA preparations. Methods aimed at the acquisition of single cells (e.g., laser microdissection techniques) frequently require a variety of additional steps that bear the risk of RNA degradation. Another problem may arise in the case of research on human tissue samples. Periods of warm ischemia between surgery and sample processing pose a serious threat to RNA integrity. It is often very challenging to decrease such times to a minimum within the framework of clinical routine procedures. RNA quality may also be impaired in samples stored for a long time or under suboptimal conditions. However, retrospective studies using the microarray technology now available on long-term archived tissues might be extremely valuable for the detection of new prognostic markers and the development of new treatments for diseases. Therefore, while it is obvious that intact RNA constitutes the best representation of the natural state of the transcriptome, there are situations in which gene expression analysis even on partially degraded RNA may be desirable. Nevertheless, little is known about the possibility of obtaining reasonable microarray data from RNA samples with impaired quality (17). A recent publication suggests an amplification method based on random priming that is shown to work remarkably well for degraded samples (18). The aim of this study was to test a wellestablished two-round IVT protocol on $20 \mathrm{ng}$ of partially degraded RNA and to assess the impact of such RNA degradation on gene expression data in comparison with high-quality RNA. 
Table 1. Real-Time Quantitative PCR Primers

\begin{tabular}{|c|c|c|c|}
\hline Gene $^{a}$ & RefSeq $^{b}$ & Forward Primer & Reverse Primer \\
\hline $\begin{array}{l}\text { EEF2 } \\
\text { NNMT }\end{array}$ & $\begin{array}{l}\text { NM_001961 } \\
\text { NM_006169 }\end{array}$ & $\begin{array}{l}\text { 5'-TCAACGAGTCCTTTGGCTTC-3' } \\
\text { 5'-TTGAAGGGAACAGAGTCAAGG-3' }\end{array}$ & $\begin{array}{l}\text { 5'-CTGCTGTTGTCGAAGGGG-3' } \\
\text { 5'-GTCACATCACACTTCAGCACC-3' }\end{array}$ \\
\hline TMSB10 & NM_021103 & 5'-CTGCCGACCAAAGAGACC- $3^{\prime}$ & 5'-GTGGCTCGTGTCCATCTTG-3' \\
\hline MET & NM_000245 & 5'-ACATTGAAATGCACAGTTGGTC-3' & 5'-ACAGGATCCACATAGGAGAATG-3' \\
\hline SARS & NM_006513 & 5'-ATGATGGACAAGGTGGAGTTT-3' & 5'-TTTCTCAGGCACAGTGATGC-3' \\
\hline$A M A C R$ & NM_014324 & 5'-GGTCATTGATGCAAATATGGTG-3' & 5'-ATCCAACATGTTCTGTCCTCG-3' \\
\hline HSD11B2 & NM_000196 & 5'-ATCACCGGCTGTGACTCTG-3' & 5'-AGGTACGCAGCTCGATGG-3' \\
\hline
\end{tabular}

Germany). $\quad$ SYBR $^{\circledR}$ Green PCR Master Mix (Applied Biosystems) was used for PCR amplification and real-time detection of PCR products. Primers (MWG Biotech) specific for different genes were designed to have a melting temperature of $60^{\circ} \mathrm{C}$ and are shown in Table 1. PCRs were carried out

Table 2. RNA Samples Hybridized to Microarrays

\begin{tabular}{|ccccc|}
\hline $\begin{array}{c}\text { Sample/ } \\
\text { Array }^{\mathrm{a}}\end{array}$ & Source & $\begin{array}{c}\text { Degradation } \\
\text { State }\end{array}$ & $\begin{array}{c}\text { Total RNA } \\
\text { Used }^{\mathrm{b}}\end{array}$ & Microarray \\
\hline TA-U & Tumor & $\mathrm{A}$ & $15 \mu \mathrm{g}$ & U133A \\
NA-U & Normal & $\mathrm{A}$ & $15 \mu \mathrm{g}$ & U133A \\
TA-F1 & Tumor & $\mathrm{A}$ & $20 \mathrm{ng}$ & Focus \\
TA-F2 & Tumor & $\mathrm{A}$ & $20 \mathrm{ng}$ & Focus \\
NA-F & Normal & $\mathrm{A}$ & $20 \mathrm{ng}$ & Focus \\
TB-F & Tumor & $\mathrm{B}$ & $20 \mathrm{ng}$ & Focus \\
NB-F & Normal & $\mathrm{B}$ & $20 \mathrm{ng}$ & Focus \\
TD-U & Tumor & D & $20 \mathrm{ng}$ & U133A \\
ND-U & Normal & D & $20 \mathrm{ng}$ & U133A \\
aThe first two letters of each sample correspond to Figure 1, the letter after \\
the dash designates the array type (U for U133A, F for Focus). \\
b15- $\mu$ R RNA samples were prepared for microarray analysis according to \\
the standard protocol, and 20-ng RNA samples were prepared for micro- \\
array analysis according to the two-round protocol. \\
\hline
\end{tabular}

passed through a 25gauge needle. Total RNA was isolated according to the manufacturer's instructions and quantified by UV absorbance at 260 nm. Quality control was performed using the RNA 6000 Pico LabChip ${ }^{\circledR}$ kit with a Model 2100 Bioanalyzer (Agilent Technologies, Waldbronn, Germany).

\section{MATERIALS AND METHODS}

\section{RNA Samples}

Tissue samples from a renal cell carcinoma (RCC) patient were obtained from the Department of Urology, University of Tübingen. The local ethical committee approved this study, and informed consent was obtained from the patient. Following nephrectomy, fragments of normal and malignant renal tissue were dissected, shock-frozen in liquid nitrogen, and stored at $-80^{\circ} \mathrm{C}$. For the isolation of RNA, the fragments were ground by mortar and pestle under liquid nitrogen, and the frozen powder was transferred immediately into TRIzOL ${ }^{\circledR}$ reagent (Invitrogen, Karlsruhe, Germany). In order to obtain RNA degraded by endogenous ribonucleases (RNases), aliquots of powdered tissue were incubated in an equal volume of phosphate-buffered saline (PBS) at $22^{\circ} \mathrm{C}$ for different time periods. TRIzOL was added to stop degradation. Samples were homogenized by being

\section{Real-Time Quantitative PCR}

RNA samples of tumor and normal tissue at different degradation states were used to synthesize single-stranded cDNAs. Reverse transcription was carried out in a reaction volume of $20 \mu \mathrm{L}$ containing $1 \mu \mathrm{g}$ total RNA, either 120 ng random hexamer primer (Amersham Biosciences, Freiburg, Germany) or $500 \mathrm{ng}$ oligo $(\mathrm{dT})_{15}$ primer (MWG Biotech, Ebersberg, Germany), 0.5 mM dNTPs (Promega, Mannheim, Germany), 10 U RNasin ${ }^{\circledR}$ (Promega), $10 \mathrm{mM}$ dithiothreitol (DTT), $200 \mathrm{U}$ SuperScript ${ }^{\mathrm{TM}}$ II reverse transcriptase (Invitrogen), and the reaction buffer supplied with the enzyme. Negative control reactions were carried out for each sample by replacing the enzyme with water. The mixture was incubated at $25^{\circ} \mathrm{C}$ for $10 \mathrm{~min}, 42^{\circ} \mathrm{C}$ for $50 \mathrm{~min}$, and $70^{\circ} \mathrm{C}$ for $15 \mathrm{~min}$.

Real-time quantitative PCR (qPCR) was performed using the ABI PRISM ${ }^{\circledR}$ 7000 Sequence Detection System (Applied Biosystems, Darmstadt, in $20 \mu \mathrm{L}$ with $300 \mathrm{nM}$ of each primer and with the following temperature profile: $50^{\circ} \mathrm{C}$ for $2 \mathrm{~min}, 95^{\circ} \mathrm{C}$ for $10 \mathrm{~min}$, and 40 cycles of $95^{\circ} \mathrm{C}$ for $15 \mathrm{~s}$ and $60^{\circ} \mathrm{C}$ for $1 \mathrm{~min}$. All samples were amplified in duplicate. Formation of undesired side products during PCR that contribute to fluorescence was excluded by melting curve analysis after PCR.

Expression differences between tumor and normal tissue samples for different genes were calculated from PCR amplification curves by relative quantification using the comparative threshold cycle $\left(\mathrm{C}_{\mathrm{T}}\right)$ method (http: //docs.appliedbiosystems.com/ pebiodocs/04303859.pdf). The housekeeping gene eukaryotic translation elongation factor 2 (EEF2) was equally expressed in both tumor and normal tissue, according to microarray data, and was therefore chosen as a reference for normalizations. The comparative $\mathrm{C}_{\mathrm{T}}$ method may be used when PCR amplification efficiencies for target and reference primer pairs are similar and close to 1 . This was verified by serially diluting cDNA samples, performing qPCR with the different primer pairs, and calculating the amplification efficiencies from the slope of the line obtained by plotting $\mathrm{C}_{\mathrm{T}}$ values versus the logarithm of relative cDNA concentrations.

\section{Microarray Analysis}

Expression analysis was performed using Affymetrix HG-U133A or HGFocus oligonucleotide microarrays (Affymetrix, High Wycombe, UK). The Focus array contains a subset of approximately 8500 sequences from the larger U133A array. The following analyses are restricted to the sequences (equals probesets) represented on both arrays. 
An overview of the samples hybridized to microarrays is given in Table 2 .

\section{Standard Protocol}

One array pair for tumor and normal RNA of the best quality that could be obtained from the tissue samples (quality A) was generated from $15 \mu \mathrm{g}$ total RNA according to the Affymetrix eukaryotic sample and array processing standard protocol (http://www.affymetrix.com/support/ downloads/manuals/expression_s2_ manual.pdf), which is based on the IVT method originally described in Reference 5.

\section{Two-Round Protocol}

We followed the protocol described in detail by Baugh et al. (10) for two rounds of IVT starting with $20 \mathrm{ng}$ total RNA at different degradation states. The first round corresponds to the Affymetrix standard protocol described above with some modifications: firststrand cDNA synthesis was performed in a reaction volume of $2 \mu \mathrm{L}, \mathrm{T} 4$ gene 32 protein (USB, Cleveland, $\mathrm{OH}$, USA) was added at a concentration of $0.4 \mu \mathrm{g} / \mu \mathrm{L}$ to increase the processivity of the reverse transcriptase (10), and the temperature was elevated to $48^{\circ} \mathrm{C}$ to increase yields. After the secondstrand synthesis, IVT was performed with nonbiotinylated NTPs using the AmpliScribe $^{\mathrm{TM}}$ T7 High Yield Tran-

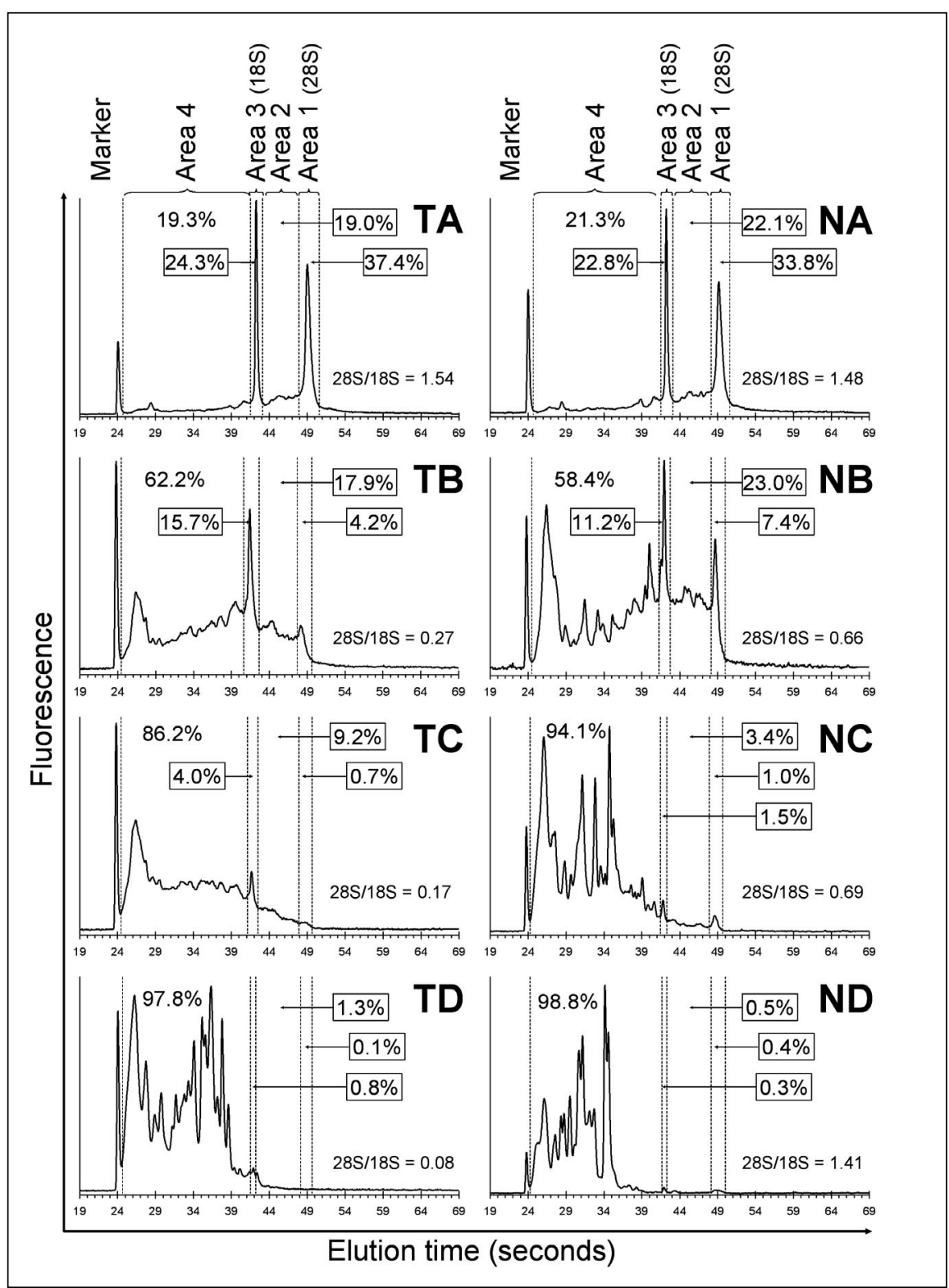

Figure 1. Electropherograms of RNA samples degraded to different extents. Analysis was performed using the RNA 6000 Pico LabChip Kit on a Bioanalyzer. The left column shows RNA samples isolated from tumor (TA-TD), the right column from normal healthy tissue (NA-ND). Tissue sample were incubated at room temperature for the following time periods prior to RNA isolation: TA, NA, $0 \mathrm{~min}$ (freshly isolated); TB, $10 \mathrm{~min}$; NB, $2 \mathrm{~min}$; TC, $20 \mathrm{~min}$; NC, $5 \mathrm{~min}$; TD, $90 \mathrm{~min}$; ND, $15 \mathrm{~min}$. The letters A, B, C, and D were assigned to samples in the order of increasing degradation. Although degradation patterns vary considerably between tumor and normal samples, the same letter was chosen for samples of comparable quality. To assess the size distribution of the RNA fragments quantitatively, four adjacent areas were defined as indicated. For an approximate calculation of the RNA amount, each intensity value (after subtraction of the baseline) was multiplied by the time between the data points, and the results were summed up for each area. Percentages given in the figure indicate RNA amounts in each area in relation to the combined amount in all four areas. $28 \mathrm{~S} / 18 \mathrm{~S}$ ratios were calculated from the respective percentages. 
scription Kit (Epicentre, Madison, WI, USA) for $9 \mathrm{~h}$ at $42^{\circ} \mathrm{C}$. First-round aRNA was cleaned using RNeasy spin columns (Qiagen, Hilden, Germany) and used to generate second-round cDNA in a random hexamer-primed reverse transcription. The second cDNA strand was synthesized using a T7(dT) 24 primer (MWG Biotech). Biotinylated aRNA was generated from this double-stranded cDNA for $8 \mathrm{~h}$ at $40^{\circ} \mathrm{C}$ using the BioArray ${ }^{\mathrm{TM}}$ HighYield ${ }^{\mathrm{TM}}$ RNA Transcript Labeling kit (Enzo Life Sciences, Farmingdale, NY, USA).

\section{Microarray Hybridization and Staining}

All procedures were carried out according to the Affymetrix standard protocol. Biotinylated aRNA was cleaned by RNeasy columns and fragmented, and $15 \mu \mathrm{g}$ were used to prepare the hybridization cocktail. After hybridization for $16 \mathrm{~h}$, microarrays were washed and stained using the instrument's standard protocol for the particular array type. Staining was performed with streptavidin-phycoerythrin using antibody-mediated signal amplification.

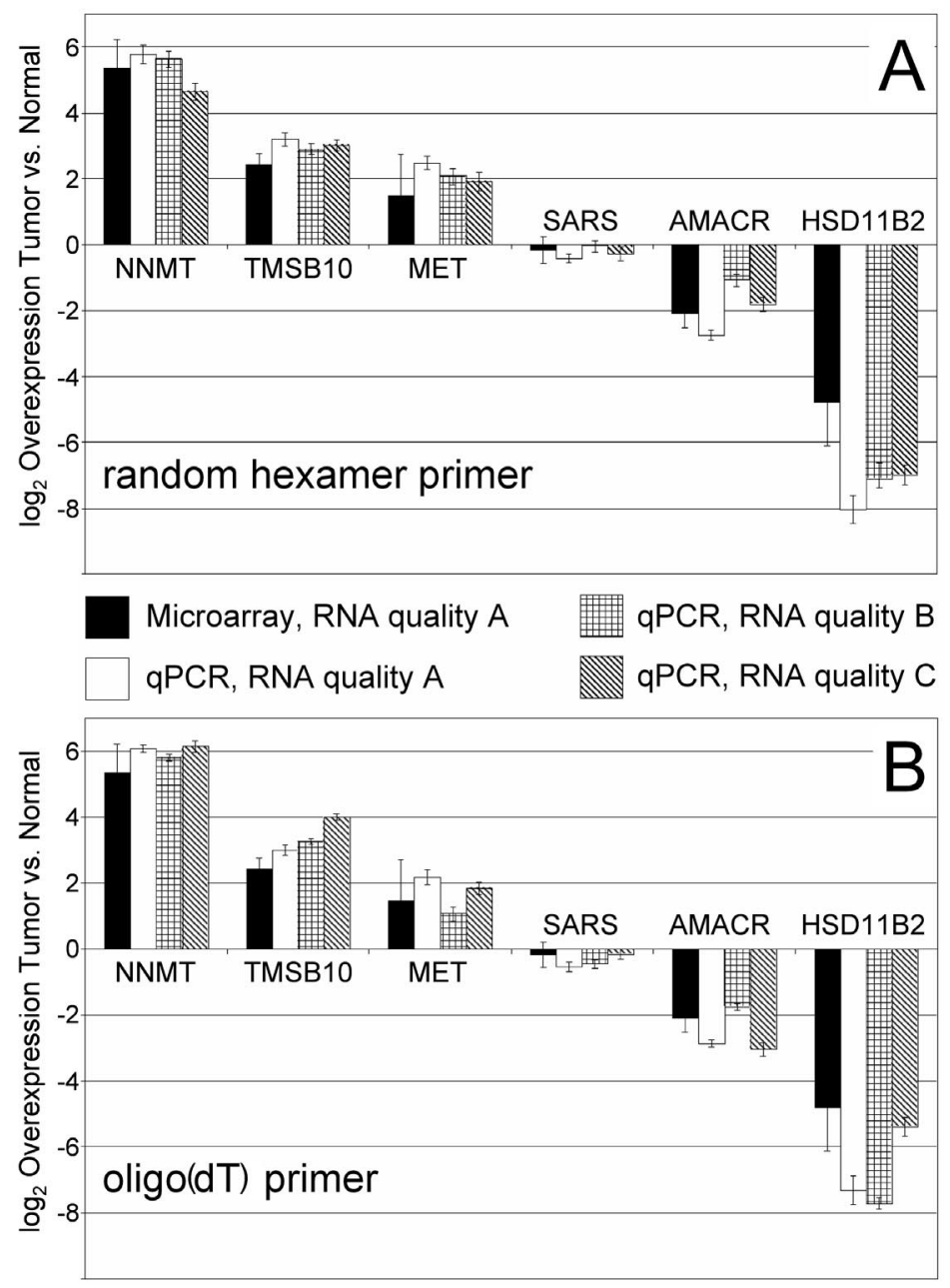

Figure 2. Differential expression of selected genes between tumor and healthy normal tissue measured by real-time quantitative PCR (qPCR) and microarrays. Negative values indicate overexpression in normal tissue compared with the tumor. (A) cDNA was synthesized using random hexamer primers, and for (B) an oligo(dT) primer was used. The first bar for each gene (Microarray, RNA quality A) indicates the expression difference measured by microarray using the samples TA-U and NA-U (see Table 2). The following three bars show qPCR measurements using cDNAs from the samples with degradation states A, B, and C (see Figure 1). qPCR values are normalized to the endogenous reference gene $E E F 2$. Error bars for $\mathrm{qPCR}$ values are standard deviations (SD). Error bars for microarray values indicate $95 \%$ confidence intervals as calculated by the MAS 5.0 software.

\section{Data Analysis}

Scanned images were processed using the Microarray Analysis Suite 5.0 (MAS 5.0; Affymetrix). Scaling of signal intensities was performed for each array based on the average intensity of 100 probesets representing housekeeping genes, which are supposed to be expressed at similar levels in different cell types and which were selected by Affymetrix (http://www.affymetrix.com/ support/technical/mask_files.affx). Expression differences between tumor and normal samples were determined by baseline comparison algorithms provided by MAS 5.0. The normal sample was always defined as the baseline. Data were further processed using Microsoft ${ }^{\circledR}$ Access and Excel ${ }^{\circledR}$.

\section{RESULTS AND DISCUSSION}

\section{RNA Degradation}

Powdered frozen tissue samples from an RCC patient were thawed and then incubated at room temperature in order to obtain RNA degraded by ribonucleases present in the tissue itself. This should lead to degradation patterns representative for naturally degraded samples. The quality of total RNA preparations is generally assessed according to the clear visibility of ribosomal $28 \mathrm{~S}$ and $18 \mathrm{~S}$ bands at a ratio of 2:1 and the absence of any other nucleic acid species after separation by electrophoresis. We performed capillary electrophoresis of high-quality and degraded samples using a Bioanalyzer (Figure 1). The incubation times necessary to reach a certain degradation state were considerably longer for the tumor as compared to the normal tissue samples. This might reflect either decreased RNase activity in this tumor tissue or some other mechanism of RNA stabilization in the tumor. The observation cannot be explained by an overexpression of ribonucleases at the mRNA level in normal tissue as indicated by the microarray data of these samples (data not shown).

For a quantitative description of the RNA degradation state, we found the $28 \mathrm{~S} / 18 \mathrm{~S}$ ratio to be of no practical value. It should only be used as long 
Table 3. Characteristics of Microarray Experiments

\begin{tabular}{|c|c|c|c|c|c|}
\hline Sample/Array ${ }^{a}$ & $\begin{array}{c}\text { aRNA Yield }{ }^{b} \\
(\mu g)\end{array}$ & Scale Factor & $\begin{array}{c}\text { Genes Present }{ }^{\mathrm{C}} \\
(\%)\end{array}$ & $\begin{array}{l}\text { 3'/5' Ratio }^{\prime} \\
\text { (GAPDH) }\end{array}$ & $\begin{array}{c}\text { 3'/5' Ratio }^{d} \\
\text { ( } \beta \text {-Actin) }\end{array}$ \\
\hline TA-U & 67 & 0.86 & 52.2 & 0.91 & 1.08 \\
\hline NA-U & 72 & 1.13 & 50.5 & 1.07 & 1.13 \\
\hline TA-F1 & 53 & 0.63 & 58.4 & 1.34 & 3.87 \\
\hline TA-F2 & 59 & 0.53 & 58.6 & 1.17 & 5.44 \\
\hline$N A-F$ & 69 & 0.68 & 56.1 & 1.91 & 6.04 \\
\hline TB-F & 23 & 0.98 & 54.1 & 3.52 & 22.82 \\
\hline NB-F & 41 & 1.07 & 55.6 & 2.79 & 9.04 \\
\hline TD-U & 11 & 10.22 & 16.1 & 9.05 & 11.46 \\
\hline ND-U & 24 & 2.01 & 36.5 & 6.94 & 13.58 \\
\hline \multicolumn{6}{|c|}{$\begin{array}{l}\text { aThe first two letters of each sample correspond to Figure } 1 \text {, the letter after the dash designates the array type (U for } \\
\text { U133A, F for Focus). } \\
\text { bAntisense RNA (aRNA) yields refer to biotinylated aRNA. For TA-U and NA-U, only } 50 \% \text { of cDNAs were used for in vitro } \\
\text { transcription (IVT). Equal amounts of } 15 \mu \mathrm{g} \text { aRNA were hybridized to microarrays with the exception of TD-U and ND-U, } \\
\text { for which only } 11 \mu \mathrm{g} \text { were used. } \\
\text { cThe percentage of genes detected as present refers to } 8746 \text { probesets represented on the Focus array. } \\
\mathrm{d}^{\prime} 3^{\prime} / 5^{\prime} \text { ratios are calculated using different probesets on the array that target the } 3^{\prime} \text { or } 5^{\prime} \text { end of the particular gene. Ratios } \\
\text { greater than } 1 \text { indicate a loss of } 5^{\prime} \text { ends among aRNA transcripts. }\end{array}$} \\
\hline
\end{tabular}

frequently used as an independent method for the verification of microarray data. Comparison of the first two bars for each gene in Figure 2 shows a high concordance between the two methods. HSD11B2 was not clearly detected above background levels in the tumor sample. Therefore, the expression difference between tumor and normal samples for this gene reflects the expression in normal tissue compared with the background of the particular method.

Expression differences obtained from degraded samples using random-primed

as there are no prominent degradation products. Otherwise, its sole use for the characterization of the sample can be misleading. This is especially apparent for the heavily degraded sample ND (Figure 1), which still has a $28 \mathrm{~S} / 18 \mathrm{~S}$ ratio of 1.4. Therefore, we decided

\begin{tabular}{|c|c|c|c|c|c|}
\hline & 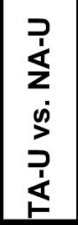 & 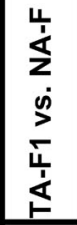 & 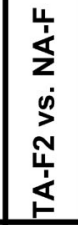 & 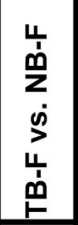 & 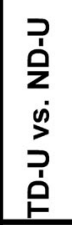 \\
\hline TA-U vs. NA-U & 1405 & $85 \%$ & $84 \%$ & $73 \%$ & $62 \%$ \\
\hline TA-F1 vs. NA-F & 1191 & 1712 & $88 \%$ & $70 \%$ & $65 \%$ \\
\hline TA-F2 vs. NA-F & 1175 & 1430 & 1626 & $70 \%$ & $63 \%$ \\
\hline TB-F vs. NB-F & 1020 & 1199 & 1134 & 1715 & $62 \%$ \\
\hline TD-U vs. ND-U & 388 & 409 & 397 & 389 & 629 \\
\hline
\end{tabular}

Figure 3. Differentially expressed genes identified in common between samples at different degradation states. Black cells show the number of genes that were identified as either increased or decreased between tumor and normal tissue by each particular pair of microarrays (see Table 2). The lower left part of each table indicates the number of such up- and down-regulated genes that were found in common between different microarray pairs. The grey shaded upper right part of the tables shows the corresponding percentages. The smaller number of regulated genes among the two array pairs constitutes the maximum number that could be detected in common and was therefore set as $100 \%$. To be considered as differentially expressed, genes had to fulfill general criteria for up- or down-regulation: an "increase" call together with a "present" call on the tumor array or a "decrease" call together with a "present" call on the normal array. Furthermore, a threshold criterion of a 2-fold increase or decrease had to be met. to include two additional parameters in the assessment: $(i)$ the amount of degradation products between the ribosomal peaks, and (ii) the amount of degradation products below the $18 \mathrm{~S}$ peak. According to this model, the RNA samples that we refer to as "moderately degraded" (TB and NB, Figure 1) can be characterized as follows: the $28 \mathrm{~S}$ peak still accounts for approximately 5\% of the overall amount of RNA. Twenty percent of fragmented RNA is located between the $28 \mathrm{~S}$ and $18 \mathrm{~S}$ rRNA, whereas a bit more than $10 \%$ is found in the $18 \mathrm{~S}$ area. Most importantly, about $60 \%$ of the overall RNA has a fragment size smaller than $18 \mathrm{~S}$ rRNA. We would regard a sample that has more than 65\% RNA in this area and less than $4 \%$ in the $28 \mathrm{~S}$ area as heavily degraded.

\section{Relative Expression Levels Measured by qPCR Are Conserved in Partially Degraded RNA}

qPCR was performed on cDNA synthesized directly from intact or degraded RNA samples without previous amplification by IVT. Figure 2 shows expression differences between tumor and normal healthy tissue for six genes measured at different RNA degradation states $\mathrm{A}, \mathrm{B}$, and $\mathrm{C}$ as defined in Figure 1. qPCR measurements are
cDNA corresponded considerably well to high-quality samples (Figure 2A). This is to be expected since random priming captures all RNA fragments, and $\mathrm{qPCR}$ products have a length in the range of only $100 \mathrm{bp}$. Therefore, fragmentation of RNA will result in a loss of the molecule for qPCR detection only if the break occurs within the short product. This might be a rare event in only moderately degraded RNA. For the cDNA samples synthesized with oligo(dT) primers, only poly(A) bearing $3^{\prime}$ ends of mRNA molecules are reverse-transcribed. In degraded samples, all fragments missing the original $3^{\prime}$ end are lost. Nevertheless, expression differences measured with oligo(dT)primed cDNA from degraded RNA (Figure 2B) still corresponded quite well to the values obtained from intact RNA. This might indicate that each individual mRNA species was affected quite uniformly by degradation in both tumor and normal tissue.

\section{Moderate RNA Degradation Disturbs Microarray Results Only Slightly}

qPCR measurements with oligo(dT)primed cDNA indicated that the $3^{\prime}$ ends of degraded mRNA were sufficient to obtain reasonable gene expression differences. This finding encouraged us to apply a protocol on degraded RNA that is capable of generating labeled target RNA for microarray analysis by two rounds of IVT from total RNA amounts 
in the low nanogram range. This protocol leads to a pronounced $3^{\prime}$ bias of the resulting antisense RNA transcripts, especially in degraded samples. Our aim was to test the impact of RNA degradation on the measurement of differential gene expression using Affymetrix microarrays that are designed to preferentially probe the $3^{\prime}$ ends of transcripts. The two-round IVT protocol was applied to $20 \mathrm{ng}$ samples of degradation states A, B, and D (Figure 1). Samples TA and NA were also subjected to the standard one-round protocol using 15 $\mu \mathrm{g}$ RNA.

Table 3 gives an overview of some general characteristics for each microarray. The two-round protocol applied to high-quality RNA (TA-F1, TA-F2, NA-F) led to lower scale factors, slightly higher percentages of genes detected as present, and increased $3^{\prime} / 5^{\prime}$ ratios if compared to the standard protocol for high-quality RNA (TA-U, NA-U). These differences may be explained by the fact that $3^{\prime}$-biased target RNA was applied to a $3^{\prime}$-biased microarray. Moderate degradation (TB-F, NB-F) caused an increase in scale factors and $3^{\prime} / 5^{\prime}$ ratios but only a slight decrease in the percentage of present genes, indicating that the sensitivity was largely unaffected by this kind of degradation. A pronounced loss in data quality became apparent for the most degraded samples (TD-U, ND-U).

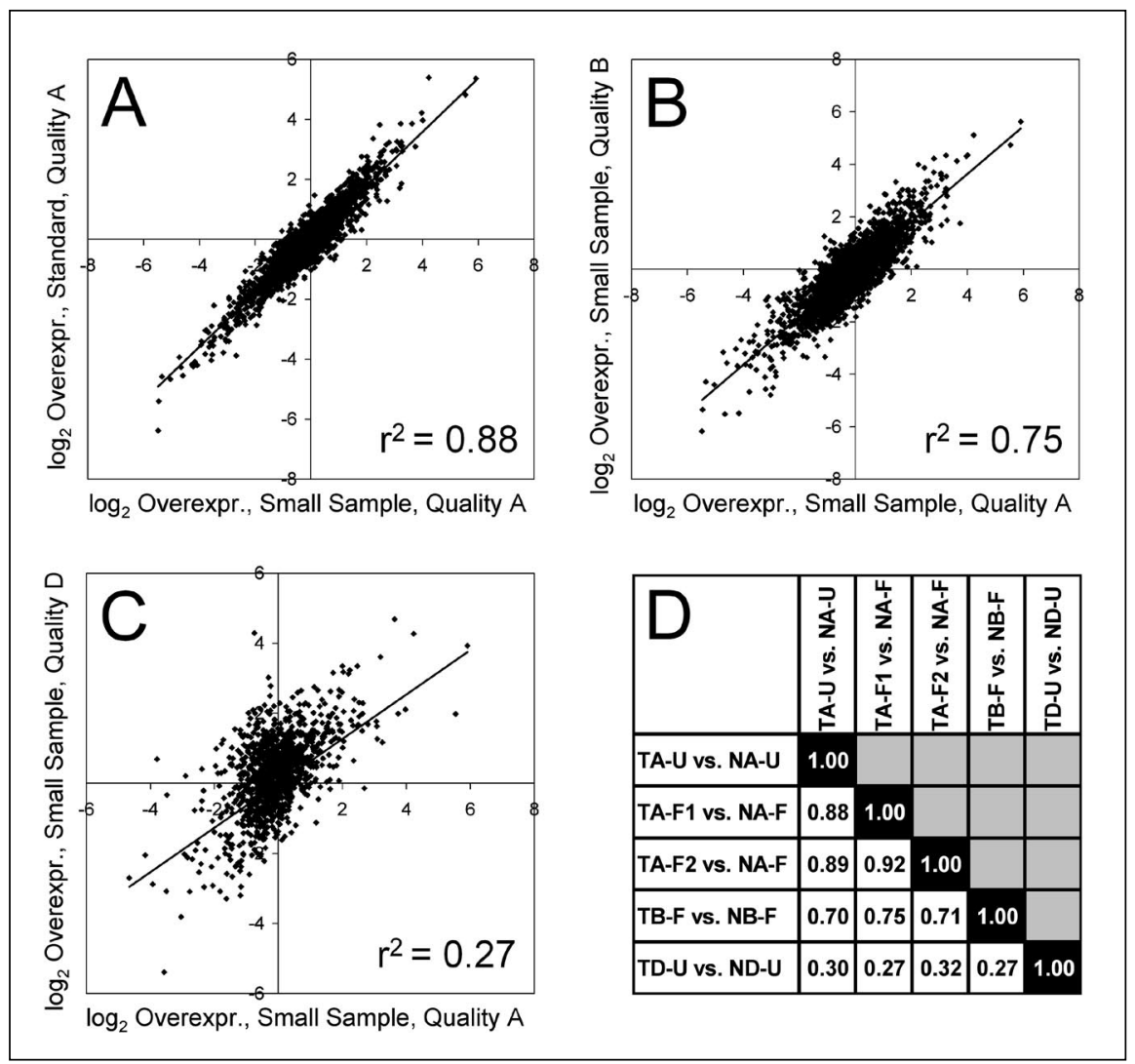

Figure 4. Correlation of overexpression measured at different degradation states. Expression differences between tumor and normal tissue were quantitatively determined by baseline comparisons using the MAS 5.0 software for different pairs of tumor and normal arrays. The $\log _{2}$ values for differential expression obtained by each array pair were compared with the other pairs. (A) Comparison of the standard labeling protocol using high-quality RNA (TA-U vs. NA-U; see Table 2) with the small sample protocol applied to the same RNA (TA-F1 vs. NA-F). (B) Partially degraded RNA (TB-F vs. NB-F) compared with high-quality RNA (TA-F1 vs. NA-F). (C) Completely degraded RNA (TD-U vs. ND-U) compared with high-quality RNA (TA-F1 vs. NA-F). (D) Correlation coefficients $r^{2}$ for all comparisons between array pairs. This analysis was restricted to 3288 genes that were detected as present on all microarrays of degradation state A and B. For comparisons that involve the most degraded samples D, the analysis had to be further restricted to 1129 genes also present on those arrays. This preselection of genes is necessary in order to avoid measuring differential expression against background levels, which would result in meaningless numbers inappropriate for measuring correlation in this way. 
In order to identify differentially expressed genes, microarrays from normal tissue samples were defined as the baseline, and tumor samples were compared to them. Figure 3 shows numbers of genes identified as up- or down-regulated in common between different pairs of tumor and normal arrays together with the corresponding percentages. The highest percentage of overlapping genes (88\%) was found for replicate tumor arrays compared with the same normal array (TA-F1 vs. NA-F compared with TA-F2 vs. NAF). This replicate setting indicates the minimal extent of inevitable variation immanent in the sample preparation and measurement procedure. The comparison of those small sample replicate pairs with the standard protocol array pair of the same starting RNA (TA-U vs. NA-U) showed slightly decreased percentages (approximately 85\%) representing small differences between the two protocols. The partially degraded sample pair (TB-F vs. NB-F), however, demonstrated a clear loss in concordance with the data obtained from high-quality samples. Whereas all high-quality samples showed an average $86 \%$ overlap regardless of the protocol used for preparation, it decreased to an average of $71 \%$ if the partially degraded pair was compared to each of the high-quality samples. For the completely degraded sample pair, the absolute number of differentially expressed genes was markedly lower than for the high-quality and partly degraded samples, which showed no differences in this parameter. This resulted in a strong decrease in the number of regulated genes identified in common with the other sample pairs. It is remarkable, that elevating or lowering the threshold for the genes to be considered for this approach does not noticeably change the results (data not shown). This indicates that the perturbation introduced by the degradation cannot be filtered out by focusing only on genes with strong expression differences.

In addition to this kind of analysis, we wanted to obtain a more comprehensive picture by analyzing differential expression in a more quantitative way. Figure 4 shows expression differences for a large set of genes measured by two different array pairs at a time.
Each data point indicates the $\log _{2}$ foldchange values for one gene measured by each of the compared array pairs. Correlation coefficients for all comparisons of tumor versus normal pairs are given in Figure 4D. As expected, the highest $r^{2}$ value (0.92) was achieved by comparing semi-replicate array pairs (TA-F1 vs. NA-F and TA-F2 vs. NA-F). Comparison of these pairs with the standard protocol pair of the same RNA (TA-U vs. NA-U) resulted in $r^{2}$ values close to the former $(0.88$ and 0.89 ). The correlation coefficients were somewhat decreased when the partially degraded sample pair was compared to the high-quality pairs $(0.70-0.75)$. However, this result still indicates a distinct tendency of this sample pair to reflect the overall expression pattern observed with high-quality samples. In contrast, the most degraded sample pair showed only a weak correlation with the other samples, which makes a trustworthy analysis impossible.

Taken together, our methods for analyzing the microarray data indicated a certain decrease in data quality for the moderately degraded samples. A distinct loss of $5^{\prime}$ ends of transcripts was evident from the $3^{\prime} / 5^{\prime}$ ratios measured by microarrays. Nevertheless, a pronounced decrease in the number of genes detected or differentially expressed did not result from this loss. However, moderate RNA degradation introduced a certain variation into differential expression data. Even though comparable in size, sets of up- or down-regulated genes between partially degraded and high-quality samples showed a smaller overlap than was observed among high-quality samples alone. Furthermore, correlations of quantitative expression differences determined between partially degraded and intact samples dropped behind correlations measured with intact RNA samples alone. Data obtained with the most degraded samples demonstrated such a low overall quality and correlation with the initial samples that they could not be reasonably considered for analysis.

Genes identified using this approach should be validated with special care. While possible perturbations introduced by RNA degradation itself cannot be accounted for, at least possible artificial influences caused by the $3^{\prime}$ bias of the two-round IVT procedure can be excluded by qPCR measurements using random-primed cDNA, which contains all mRNA fragments of the degraded sample.

In conclusion, while all efforts should be made to obtain high-quality RNA samples that reflect the natural state most reliably, moderately degraded samples with a degradation signature similar to our samples TB and NB (Figure 1) $-5 \%$ of the RNA in the 28S rRNA area and no more than $60 \%$ with fragment sizes smaller than $18 \mathrm{~S}$ rRNA - may still lead to a reasonable expression profile. Twenty nanograms of such degraded RNA are sufficient for microarray analysis after two rounds of linear amplification by IVT. Expression differences measured with this method and verified by real-time qPCR are similar to those obtained from high-quality samples.

\section{ACKNOWLEDGMENTS}

We would like to thank Sven Poths and Dr. Michael Bonin of the Microarray Facility Tübingen for their excellent technical support and Lynne Yakes for help with preparation of the manuscript. This work was supported by a grant from the German Federal Ministry of Education and Research (Fö. 01KS9602) and the Interdisciplinary Center of Clinical Research Tübingen (IZKF, Project S.04.00088).

\section{REFERENCES}

\footnotetext{
1.Panda, S., T.K. Sato, G.M. Hampton, and J.B. Hogenesch. 2003. An array of insights: application of DNA chip technology in the study of cell biology. Trends Cell Biol. 13: 151-156.

2.Sevenet, N. and O. Cussenot. 2003. DNA microarrays in clinical practice: past, present, and future. Clin. Exp. Med. 3:1-3.

3.Iscove, N.N., M. Barbara, M. Gu, M. Gibson, C. Modi, and N. Winegarden. 2002. Representation is faithfully preserved in global cDNA amplified exponentially from sub-picogram quantities of mRNA. Nat. Biotechnol. 20:940-943.

4.Klein, C.A., S. Seidl, K. Petat-Dutter, S. Offner, J.B. Geigl, O. Schmidt-Kittler, N. Wendler, B. Passlick, et al. 2002. Combined transcriptome and genome analysis of single micrometastatic cells. Nat. Biotechnol. 20: 387-392.
} 
5.Van Gelder, R.N., M.E. von Zastrow, A. Yool, W.C. Dement, J.D. Barchas, and J.H. Eberwine. 1990. Amplified RNA synthesized from limited quantities of heterogeneous cDNA. Proc. Natl. Acad. Sci. USA 87:16631667.

6.Luo, L., R.C. Salunga, H. Guo, A. Bittner, K.C. Joy, J.E. Galindo, H. Xiao, K.E. Rogers, et al. 1999. Gene expression profiles of laser-captured adjacent neuronal subtypes. Nat. Med. 5:117-122.

7.Ohyama, H., X. Zhang, Y. Kohno, I. Alevizos, M. Posner, D.T. Wong, and R. Todd. 2000. Laser capture microdissection-generated target sample for high-density oligonucleotide array hybridization. BioTechniques 29:530-536.

8.Luzzi, V., V. Holtschlag, and M.A. Watson. 2001. Expression profiling of ductal carcinoma in situ by laser capture microdissection and high-density oligonucleotide arrays. Am. J. Pathol. 158:2005-2010.

9.Scheidl, S.J., S. Nilsson, M. Kalen, M. Hellstrom, M. Takemoto, J. Hakansson, and $P$. Lindahl. 2002. mRNA expression profiling of laser microbeam microdissected cells from slender embryonic structures. Am. J. Pathol. 160:801-813.

10.Baugh, L.R., A.A. Hill, E.L. Brown, and C.P. Hunter. 2001. Quantitative analysis of mRNA amplification by in vitro transcription.
Nucleic Acids Res. 29:E29.

11.Feldman, A.L., N.G. Costouros, E. Wang, M. Qian, F.M. Marincola, H.R. Alexander, and S.K. Libutti. 2002. Advantages of mRNA amplification for microarray analysis. BioTechniques 33:906-914.

12.Zhao, H., T. Hastie, M.L. Whitfield, A.L. Borresen-Dale, and S.S. Jeffrey. 2002. Optimization and evaluation of T7 based RNA linear amplification protocols for cDNA microarray analysis. BMC Genomics 3:31.

13.Luzzi, V., M. Mahadevappa, R. Raja, J.A. Warrington, and M.A. Watson. 2003. Accurate and reproducible gene expression profiles from laser capture microdissection, transcript amplification, and high density oligonucleotide microarray analysis. J. Mol. Diagn. 5:9-14.

14.McClintick, J.N., R.E. Jerome, C.R. Nicholson, D.W. Crabb, and H.J. Edenberg. 2003. Reproducibility of oligonucleotide arrays using small samples. BMC Genomics 4:4.

15.Nygaard, V., A. Loland, M. Holden, M. Langaas, H. Rue, F. Liu, O. Myklebost, O. Fodstad, et al. 2003. Effects of mRNA amplification on gene expression ratios in cDNA experiments estimated by analysis of variance. BMC Genomics 4:11.

16.Wang, J., L. Hu, S.R. Hamilton, K.R. Coombes, and W. Zhang. 2003. RNA amplification strategies for cDNA microarray experiments. BioTechniques 34:394-400.

17.Dash, A., I.P. Maine, S. Varambally, R. Shen, A.M. Chinnaiyan, and M.A. Rubin. 2002. Changes in differential gene expression because of warm ischemia time of radical prostatectomy specimens. Am. J. Pathol. 161: 1743-1748.

18.Xiang, C.C., M. Chen, L. Ma, Q.N. Phan, J.M. Inman, O.A. Kozhich, and M.J. Brownstein. 2003. A new strategy to amplify degraded RNA from small tissue samples for microarray studies. Nucleic Acids Res. 31: e53.

Received 05 August 2003; accepted 15 September 2003.

Address correspondence to:

Stefan Stevanović

University of Tübingen

Institute for Cell Biology, Department of Immunology

Auf der Morgenstelle 15

D-72076 Tübingen, Germany

e-mail:stefan.stevanovic@uni-tuebingen.de 